\title{
Combination diuretic treatment in severe heart failure: a randomised controlled trial
}

\author{
K S Channer, K A McLean, P Lawson-Matthew, M Richardson
}

Department of

Hallamshire Hospital,

Sheffield

K S Channer

K A McLean

P Lawson-Matthew

Department of

Geriatrics, South

Tyneside General

Hospital, Southshields,

Tyne and Wear

M Richardson

Correspondence to:

Dr K S Channer,

Department of Cardiology,

Royal Hallamshire Hospital,

Glossop Road, Sheffield

S10 2JF.

Accepted for publication 15 September 1993

\begin{abstract}
Objectives-(a) To test the hypothesis that a fixed 3 day course of the combination of a thiazide and loop diuretic is as effective as more prolonged treatment in the management of severe resistant cardiac failure. (b) To compare two thiazide diuretics (bendrofluazide and metolazone) in combination with loop diuretics in the treatment of severe resistant cardiac failure.
\end{abstract}

Design-Randomised study with a $2 \times 2$ factorial design.

Setting-Provincial teaching hospital.

Patients-33 consecutive patients (40 episodes) admitted with severe congestive cardiac failure (New York Heart Association class III or IV) unresponsive to intravenous loop diuretics for 48 hours.

Main outcome measures-Change in daily weight and serum electrolytes and clinical improvement in heart failure.

Results-Diuresis was established during 37 of 40 episodes; of the rest two patients died in hospital. On 36 occasions improvement was sufficient to allow discharge from hospital. Median (range) maximal weight loss was $-5.05(-11.3$ to $1.6) \mathrm{kg}$ after the addition of bendrofluazide and $-5.6(-12.2$ to $4 \cdot 8) \mathrm{kg}$ after the addition of metolazone (NS). Area under the body weight loss against time curves showed no significant difference between the two thiazide diuretics. Median (range) maximal weight loss after three days of treatment was $-5 \cdot 4(-12 \cdot 2$ to $4 \cdot 8)$ $\mathrm{kg}$ and $-5.5(-10.3$ to 1$) \mathrm{kg}$ after a more prolonged course of median (range) 5.6 (1 to 13) days (NS). Area under the body weight loss time curves showed no significant difference between the two durations of treatment. Bendrofluazide was associated with fewer electrolyte disturbances.

Conclusions-Bendrofluazide and metolazone were equally effective in establishing a diuresis in patients with severe congestive cardiac failure resistant to loop diuretics. A fixed three day course of the combination was as effective as a longer course.

(Br Heart f 1994;71:146-150)

Severe heart failure carries a poor prognosis with up to $50 \%$ mortality within 12 months. ${ }^{1}$
Most patients are symptomatic with dyspnoea on minimal exertion for example, washing and dressing (New York Heart Association (NYHA) class III) or at rest (NYHA class IV), and many have fluid retention with oedema and ascites. Symptomatic improvement can be achieved with diuretics but prognosis has only been shown to be improved with angiotensin conventing enzyme (ACE) inhibitors ${ }^{1}$ and vasodilators. ${ }^{23}$ Some patients become resistant to loop diuretics despite intravenous dosage, and other studies have shown a pronounced diuretic effect of the combination of a thiazide and loop diuretics. ${ }^{4-8}$

Traditionally metolazone has been used, although uncontrolled data have shown similar effects with bendrofluazide. ${ }^{8}$ There is no theoretical advantage of one thiazide over another and no comparative trials have been performed. The finding that once the diuresis has been established it is often possible to withdraw the thiazide diuretic and diuresis will continue with loop diuretics alone ${ }^{8}$ has not been tested.

In this study we used a $2 \times 2$ factorial design to test the hypothesis that short term treatment with the combination of thiazide and loop diuretic was as effective as more prolonged treatment and compared bendrofluazide with metolazone in patients with severe resistant heart failure.

\section{Patients and methods} PATIENTS

Thirty three consecutive patients $(21$ males; 12 females) mean (range) age 66 (17-86) years took part in this randomised controlled trial. Seven patients were randomised twice after readmission with recurrence of severe heart failure; there were therefore, 40 episodes of treatment. All patients gave informed consent and the study was approved by the Hospital Ethics Committee. All patients were inpatients because of severe heart failure (NYHA III/IV) and had signs of fluid retention. On admission, patients were initially treated with at least $80 \mathrm{mg}$ twice daily of frusemide by intravenous bolus. Before randomisation to the addition of a thiazide diuretic, all had failed to respond to loop diuretics and body weight was stable or had increased for at least two days, so these patients had resistant oedema.

Patients were randomised to receive either bendrofluazide $(10 \mathrm{mg})$ or metolazone (10 $\mathrm{mg}$ ), once daily orally for either three days or 
indefinitely (left to the clinician's discretion). We used a $2 \times 2$ factorial design so that 10 patients received bendrofluazide for three days and 10 patients received metolazone for three days. Similarly, 10 patients received bendrofluazide and 10 patients metolazone for a variable duration. Thus 20 patients had a fixed course and 20 patients a variable course of thiazide diuretic, 20 patients had bendrofluazide, and 20 had metolazone.

\section{MEASUREMENTS}

Efficacy of diuresis was assessed daily by weight and weight loss was judged to indicate diuresis. Weighing was performed on the same scales and at the same time each day. As often as possible patients were weighed in the same clothes. The scales used were SECA electronic digital scales which have a self test and automatic zero resetting. Repeat measurements over a five minute period were identical.

Concomitant daily serum electrolyte and urea measurements were made with the Olympus AU5200 analyser. Coefficients of variation for sodium and potassium were $<2 \%$ and for urea and creatinine were $<3 \%$. Potassium supplements in the form of effervescent potassium tablets containing 6.7 mmol potassium chloride were given when serum potassium was $<3.5 \mathrm{mmol} / 1$. All other medication was left unchanged during the study.

\section{STATISTICAL ANALYSIS}

Changes in body weight, serum electrolytes, urea, and creatinine with time were analysed by area under the curve analysis with the trapezoidal rule as recommended by Matthews et al for the analysis of serial measurements. ${ }^{9}$ The significance of the changes between groups was assessed by the Mann-Whitney $U$ test with a probability of $<0.05$ accepted as significant. The $95 \%$ confidence intervals ( $95 \% \mathrm{CIs}$ ) are quoted for the differences.

\section{Results}

Forty treatment periods were included in the study. There was balanced randomisation so that 10 patients had fixed courses of bendrofluazide and metolazone and 10 patients had variable courses of bendrofluazide and metolazone. The treatment of a loop diuretic in combination with a thiazide was well tolerated and established a diuresis and weight loss within 24 hours on 35 occasions and within 48 hours on two occasions. On three occasions no weight change was found. Four patients died in hospital (two of whom had no weight change) and the remaining patients left hospital alive with no clinical evidence of fluid retention. Thirteen were, however, readmitted with severe heart failure and 14 patients died during a follow up period of 48 months.

The results are presented for 20 treatments with bendrofluazide, 20 treatments with metolazone, 20 fixed courses, and 20 variable courses. The average (range) duration of treatment when left to the discretion of the physician was $5.6(1-13)$ days in the variable treatment group.

\section{WEIGHT LOSS}

Figure 1 shows the changes in body weight after treatment with thiazide diuretics. There were no significant differences between the baseline weight of the groups. These were bendrofluazide mean (SD) $61.4(14.7) \mathrm{kg}$, metolazone $70.8(17 \cdot 3) \mathrm{kg}$, variable course $66 \cdot 0(20 \cdot 3) \mathrm{kg}$, and fixed course $66 \cdot 2(12 \cdot 3)$ kg. Mean body weight actually increased before the introduction of the thiazide. Weight loss continued for five to six days in both the fixed and variable duration groups. There were no significant differences in the area under the curve of the weight loss against time curves when either the bendrofluazide and metolazone groups were compared or when the fixed and variable duration groups were compared. Median (range) maximum weight change was $5.05(-11.3$ to 1.6$) \mathrm{kg}$ for bendrofluazide and $-5 \cdot 6(-12 \cdot 2$ to $4 \cdot 8) \mathrm{kg}$ for metolazone (NS), and $-5 \cdot 4(-12 \cdot 2$ to $4 \cdot 8) \mathrm{kg}$ for fixed and $-5.5(-10.3$ to 1$) \mathrm{kg}$ for variable duration of treatment (NS). After the end of thiazide treatment weight increased slightly but subsequently remained stable. Changes in fluid balance were managed by alteration of the
Figure 1 Changes in body weight during treatment with bendrofluazide and metolazone given for either a fixed period of 3 days a fixed or for a duration determined clinically (mean 5.6 days; variable).

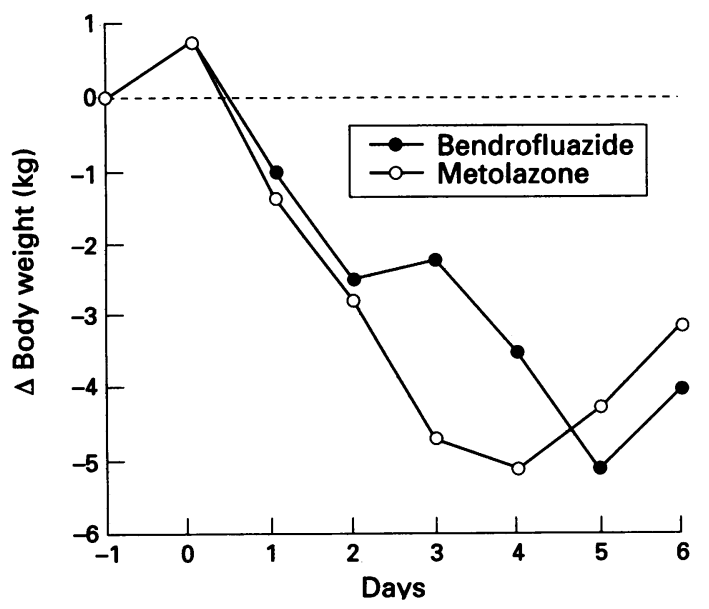


Figure 2 Changes in serum sodium during treatment with either bendrofluazide or metolazone given for either a fixed period of 3 days (fixed) or for a duration determined clinically (variable).

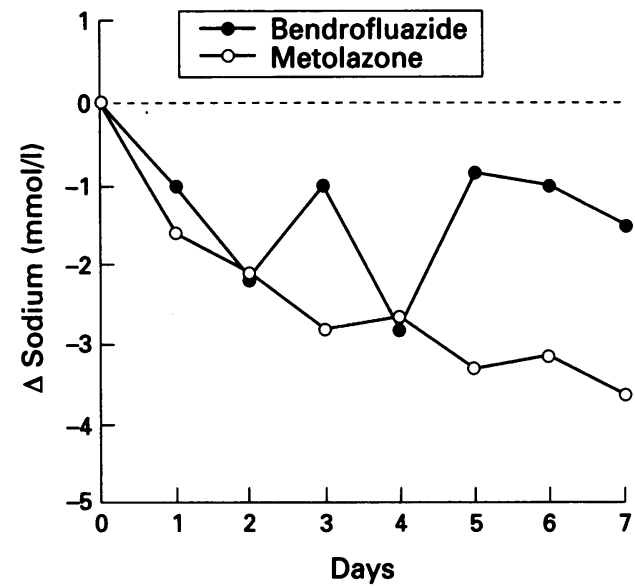

dosage of loop diuretic. All patients were discharged on only maintenance treatment of oral loop diuretic.

\section{CHANGES IN ELECTROLYTES}

Sodium

Figure 2 shows that mean serum sodium fell slightly in all groups. No significant differences were found between the fixed and variable duration groups (median area under the curve -3.5 variable, -3.75 fixed; $95 \% \mathrm{CI}$ for the difference -9.0 to $18.5, p=0.6$ ). Metolazone caused a greater change in serum sodium but this was not significantly different from that caused by bendrofluazide treatment (median area under the curve metolazone $-10 \cdot 27,-1.75$ bendrofluazide; $95 \% \mathrm{CI}$ for the difference $-19 \cdot 5$ to $6 \mathrm{p}=0.2$ ).

\section{Potassium}

Figure 3 shows that serum potassium fell with thiazide treatment in all groups. There was no difference in the change in serum potassium in the variable and fixed duration groups (median area under the curve 1.0 variable, 1.03 fixed; $95 \% \mathrm{CI}$ for difference -2.5 to $1.8, p=0.8)$. Metolazone caused a greater change in serum potassium than bendrofluazide (median area under the curve 1.93 metolazone; 0.73 bendrofluazide; $95 \%$ CI for the difference -0.55 to 3.35 , $\mathrm{p}=0 \cdot 14)$.

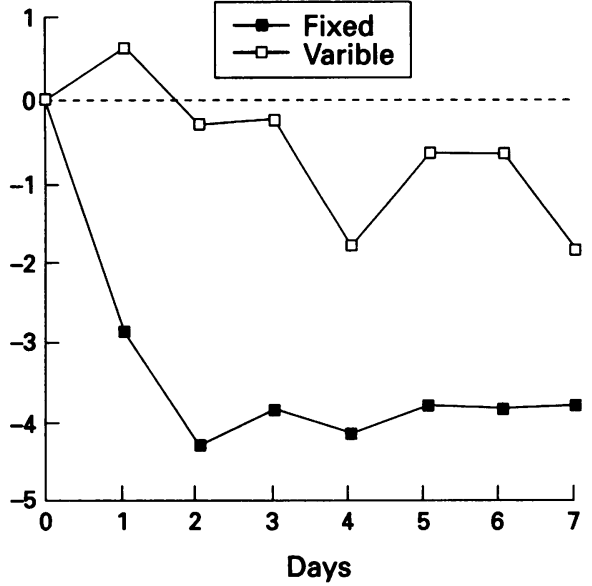

Fifteen patients developed hypokalaemia (serum potassium $<3.5 \mathrm{mmol} / \mathrm{l}$ ) during treatment with metolazone compared with 11 with bendrofluazide (NS, $\chi^{2}$ test) and 14 during variable duration compared with 13 during a fixed duration. Significantly fewer potassium supplements were given to patients taking a fixed course of thiazide than a variable course (median (range) 0 (0 to 32) fixed, 16 (0 to 112) variable, $\mathrm{p}<0.05,95 \% \mathrm{CI}$ for the difference -21 to 0.01 ). No significant difference was found between the number of potassium supplements required for patients treated with bendrofluazide or metolazone.

UREA

Figure 4 shows that blood urea rose in all groups after treatment with thiazide. There were no significant differences between fixed or variable duration (median area under the curve 4.8 variable, 9.9 fixed; $95 \% \mathrm{CI}$ for the difference -13.05 to $0.05, p=0.05$ ). Metolazone caused greater but non-significant changes in blood urea compared with bendrofluazide (median area under the curve 7.88 metolazone, 4.97 bendrofluazide; $95 \%$ $\mathrm{CI}$ for the difference -1.8 to $11.85, \mathrm{p}=$ $0 \cdot 27$ ).

\section{Creatinine}

Figure 5 shows that serum creatinine initially fell then rose in all groups. No significant dif-
Figure 3 Changes in serum potassium during treatment with bendrofluazide or metolazone given for either a fixed period of three days (fixed) or for a duration determined clinically (variable).

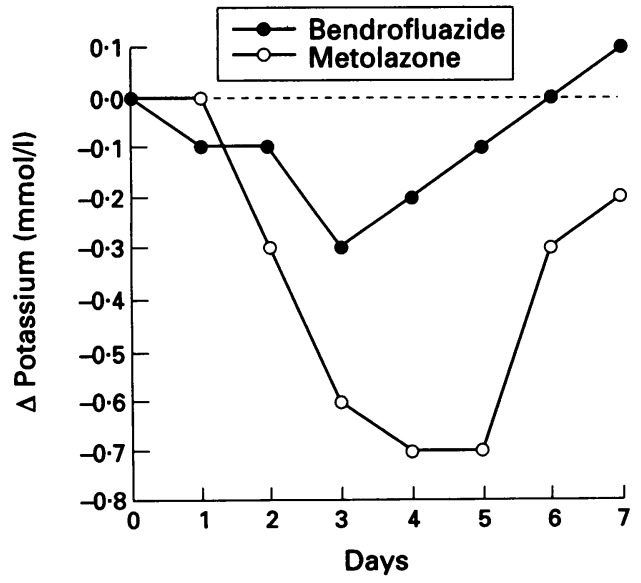


Figure 4 Changes in blood urea during treatment with bendrofluazide or metolazone given for either a fixed period of three days (fixed) or for a duration (variable). determined clinically

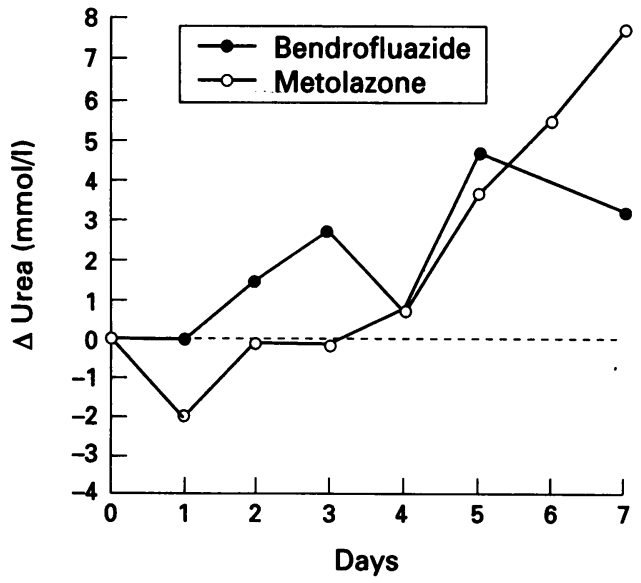

Figure 5 Changes in serum creatinine during treatment with bendrofluazide or metolazone given for either a fixed period of three days determined clinically (variable). (fixed) or for a duration

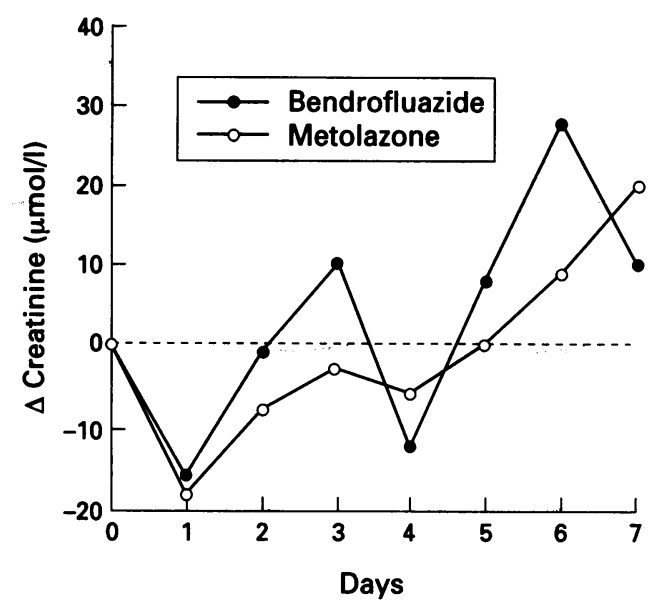

ferences were found between variable and fixed duration groups (median area under the curve 20 variable, 48.3 fixed; $95 \%$ CI for the difference -84.5 to $13.5, p=1.7$ ). Metolazone caused a significantly greater change in creatinine than did bendrofluazide (median area under the curve 44.5 metolazone, -19.25 bendrofluazide; $95 \% \mathrm{CI}$ for the difference 1.0 to $90.5, \mathrm{p}<0.05$ ).

\section{CAUSE OF HEART FAIIURE}

Most patients (21) had ischaemic heart disease, 12 had valvar heart disease, 11 had dilated cardiomyopathy, three had cor pulmonale, and two had hypertensive heart disease. Ten patients had two causes for heart failure.

\section{CONCOMITANT MEDICATION}

Potassium sparing diuretics were also prescribed in 19 patients, ACE inhibitors in 24, oral nitrates in 25 , digoxin in 18 , and oral anticoagulants in 10 . The table shows the distribution of concomitant medication in the difference arms of the study.

Table Number of patients in each treatment group taking different medication

\begin{tabular}{lllccl}
\hline Treatment & ACEI & Anticoag & Digoxin & Potspare & Nitrate \\
\hline Metolazone & 13 & 6 & 8 & 10 & 14 \\
Bendrofluazide & 11 & 4 & 10 & 9 & 11 \\
Fixed & 11 & 4 & 7 & 12 & 13 \\
Variable & 13 & 6 & 11 & 7 & 12
\end{tabular}

ACEI, angiotensin converting enzyme inhibitor; Anticoag, anticoagulant; Potspare, potassium sparing diuretic.
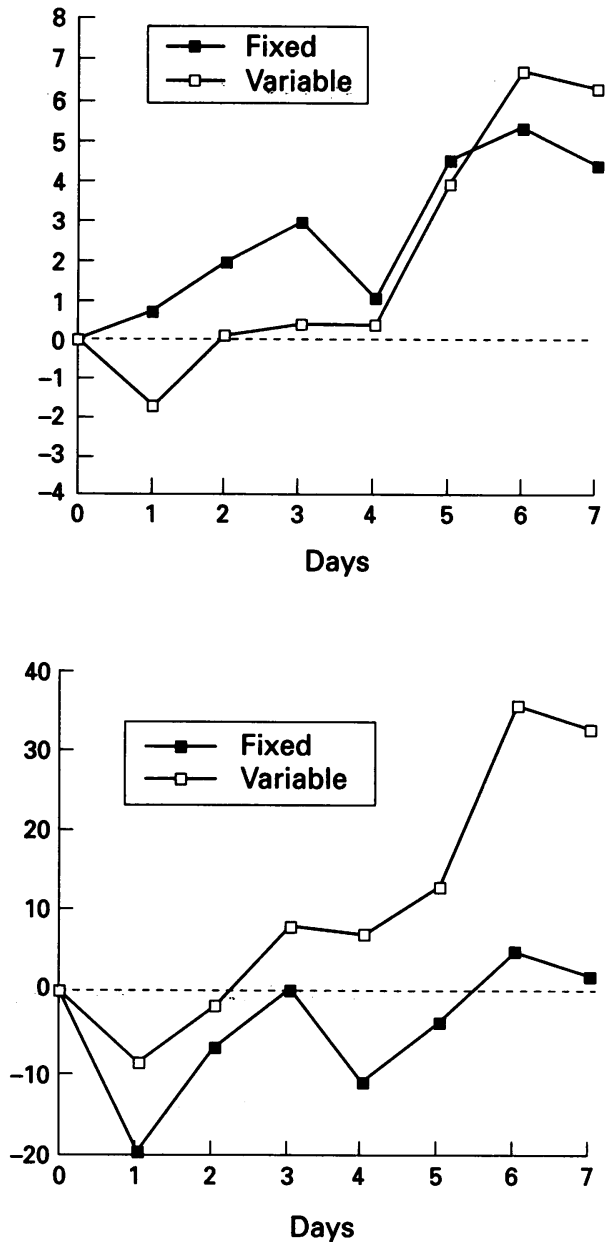

Discussion

This study shows that in patients with severe heart failure and resistant oedema, the addition of a thiazide diuretic to treatment with loop diuretics established a diuresis in most cases. The patients in this study had a poor prognosis with a $>50 \%$ short term mortality signifying the severity of their heart failure. Nevertheless, symptomatic improvement was achieved in $90 \%$ of patients, allowing their discharge from hospital. Failure to respond to the addition of a thiazide indicated a poor prognosis. This has previously been shown by Kiyingi et al. ${ }^{7}$

We have shown that both the thiazide diuretics metolazone and bendrofluazide effectively induce diuresis as we had suggested from previous uncontrolled observations. ${ }^{8}$ Electrolyte changes were, however, greater with metolazone than bendrofluazide and metolazone is 10 times more expensive than bendrofluazide at 1992 prices. More importantly, however, we have shown that once the diuresis has become established the thiazide can be safely withdrawn and diuresis will continue. As far as we are aware, this has not been reported previously. Kiyingi et al used low doses of metolazone arguing that these would avoid electrolytic disturbances. ${ }^{7}$ We used fixed high doses of $10 \mathrm{mg}$ of both thiazides to avoid the compounding effect of variable dose as well as variable duration. We believe smaller doses may initiate a diuresis in this setting but have not investigated this. Although electrolytic changes occurred they 
were rarely clinically significant. We expected potassium depletion to occur once diuresis was established. Therefore, we monitored for it and treated it with an oral potassium supplement. Although hypokalaemia occurred equally commonly during fixed and variable courses of a thiazide diuretic, fewer potassium supplements were required after a fixed course. We measured changes in serum potassium only and a more accurate assessment of the change in potassium would have been obtained by measuring total body potassium. None of these very sick patients had any complications from electrolytic changes, which were generally small.

As ACE inhibitors have been shown to prolong survival in patients with severe heart failure, all of our patients were also taking these drugs except those with contraindications or those unable to tolerate them. The groups were well matched for concomitant medication that may have influenced the diuresis. The temporary addition of a thiazide diuretic to treatment with ACE inhibitor drugs caused no specific problems.

In conclusion, in patients with severe resistant heart failure the addition of a thiazide diuretic to treatment with loop diuretics will usually establish a diuresis. Bendrofluazide and metolazone are both effective in this and treatment may only be necessary for 3 days.

1 Consensus Trial Study Group. Effects of enalapril on mortality in severe congestive heart failure. $N$ Engl $f$ Med 1987;316:1429-35.

2 Cohn IN, Archibald DG, Ziesche S, et al. Effect of vasodilator therapy on mortality in chronic congestive vasodilator therapy on mortality in chronic congestive
heart failure; results of a veterans administration coheart failure; results of a veterans administration

3 Cohn JA, Johnson G, Ziesche S, et al. A comparison of enalapril with hydralazine-isosorbide dinitrate in the treatment of chronic congestive heart failure. $N$ Engl $\mathcal{F}$ Med 1991;325:303-10.

4 Grosskopt I, Rabinovitz M, Rosenfeld IB. Combination of furosemide and metolazone in the treatment of severe congestive heart failure. Isr $\mathcal{F} \mathrm{Med} S \mathrm{Si}$ 1986;22:787-90.

5 Ghose RR, Gupta SK. Synergistic action of metolazone with 'loop' diuretics. BMF 1981;812:1432-3.

6 Gunstone RF, Wing AJ, Shani HGP, Njemo D, Sabuka EMW. Clinical experience with metolazone in 52 African patients; synergy with furosemide. Postgrad Med African patients; $\$$ sy

7 Kiyingi A, Field MJ, Pawsey CC, Yiannikas J, Lawrence JR, Arter WJ. Metolazone in treatment of severe refractory congestive cardiac failure. Lancet 1990;335:29-31.

8 Channer KS, Richardson M, Crook R, Jones JV. Thiazides with loop diuretics for severe congestive heart Thiazides with loop diuretics for

9 Matthews JNS, Altman DG, Campbell MJ, Royston P. Analysis of serial measurements in medical research. BMF 1990;300:230-5. 